WC1.3

9:45 am - 10:00 am

\title{
Exact, agile, optical frequency synthesis using an optical comb generator and optical injection phase lock loop
}

\author{
C. C. Renaud, C. F. C. Silva, M. Dueser, P. Bayvel and A. J. Seeds \\ Dept. of Electronic \& Electrical Eng., University College London, Torrington Place, London, WC1E 7JE, United Kingdom. \\ Tel: +44 (0)207679 7928, Fax: +44(0)2073889325,email:a.seeds@ee.ucl.ac.uk
}

\begin{abstract}
A novel optical source for use in agile dense wavelength division multiplexing (DWDM) networks is described. This source combines reference limited stability, wide tuning range, high spectral purity, narrow line width and fast wavelength switching.

Key words: tuneable laser; optical communications, optical injection phase lock loop, optical frequency comb generator.
\end{abstract}

Simple techniques for exact channel frequency synthesis are of considerable interest for the development and testing of high spectral efficiency dense wavelength division multiplexed (DWDM) networks [1].

This paper presents a new DWDM synthesiser system which has channel-frequency exactly determined by supplied optical and microwave reference frequencies so that DWDM guard bands (typically $3 \mathrm{GHz}-5 \mathrm{GHz}$ ) can be completely eliminated. Channel-lasers (SG-DBR or SSG-DBR) are incorporated within optical-injection phase-lock loop (OIPLL, Fig.1) blocks [2], phase-locked to output lines from an optical-frequency comb-generator (OFCG, Fig. $1,10 \mathrm{GHz})[3]$. The comb line spacing is exactly equal to the microwave reference frequency $(10 \mathrm{GHz}$ in these experiments), while the exact optical frequency of each comb line is determined by the optical reference source. The OIPLL combines the rapid acquisition and linewidth tolerance of optical injection locking with the large tracking ability of the optical phase lock loop. The combined technique also eliminates the requirement for extremely short loop propagation delay of optical phase lock loops when used with wide $(\mathrm{MHz})$ linewidth semiconductor lasers.

OFCG OIPLL

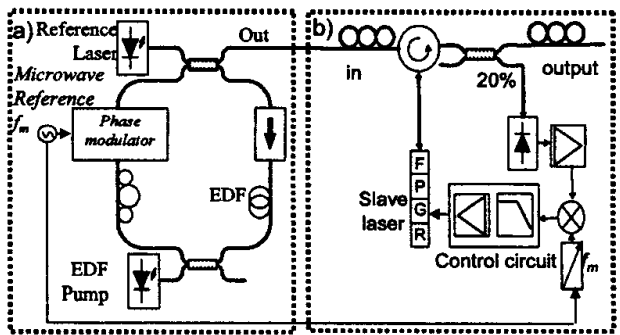

Fig. 1: Experimental optical frequency comb generator (OFCG, a) and optical injection phase lock loop (OIPLL) with a four-section tuneable laser (b).
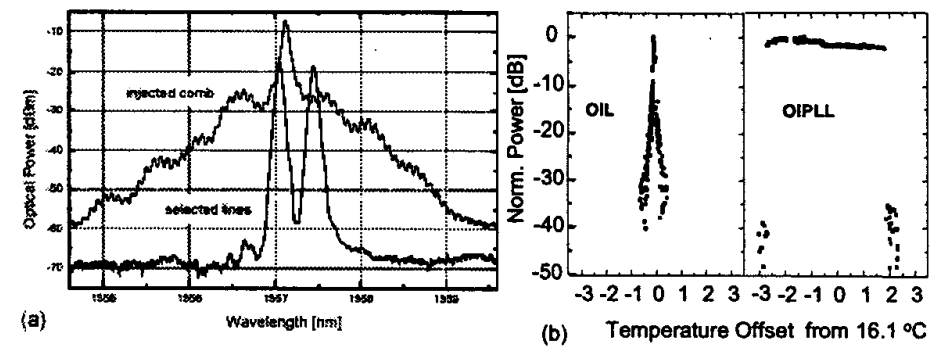

Fig. 2: a) Comb generator spectrum and output spectrum from two OIPLLs locked to channels spaced by $50 \mathrm{GHz}, \mathrm{b}$ ) Detected heterodyne signal from two OIPLL (or OIL) locked laser diodes (channel spacing $50 \mathrm{GHz}$ ).

Figure 2a. shows the output spectrum from the comb generator and the spectrum from two OIPLLs locked to comb lines separated by $50 \mathrm{GHz}$. Unwanted comb lines are seen to be suppressed by $>40 \mathrm{~dB}$ below the wanted output over a $>1.8 \mathrm{THz}$ range. By heterodyning the output from the two lasers on a high-speed photodetector and analysing the resultant signal in an electrical spectrum analyzer, the accuracy of the OIPLL locking could be investigated. Figure $2 \mathrm{~b}$. shows the detected heterodyne output power measured within a $1 \mathrm{kHz}$ bandwidth centered 
on $50 \mathrm{GHz}$, as the temperature of one channel laser was varied. This proved the quality of the locking over the temperature range, since we obtained an inter-channel frequency error $<1 \mathrm{kHz}$ (electrical spectrum analyzer resolution limited). Furthermore, the $5 \mathrm{~K}$ temperature range is equivalent to an $80 \mathrm{GHz}$ locking range, compared with $<2 \mathrm{GHz}$ when only the optical injection locking technique is used.

The system was then tested for wavelength switching by applying a current pulse to the rear grating section of the slave laser, corresponding to a wavelength jump between $1,570 \mathrm{~nm}$ and 1,532 nm. The tuning transient was measured using a Fabry-Perot interferometer scanned by a precision D/A converter under computer control. For such hopping at a frequency of $100 \mathrm{~Hz}$, the new wavelength is acquired to the system measurement accuracy of $<500 \mathrm{MHz}$, within $<5 \mu \mathrm{s}$, limited by the speed of the laser current controller, and there was no measurable long-term drift under active-locking (Fig.3). The optical output power stabilises within $15 \mu$ s.
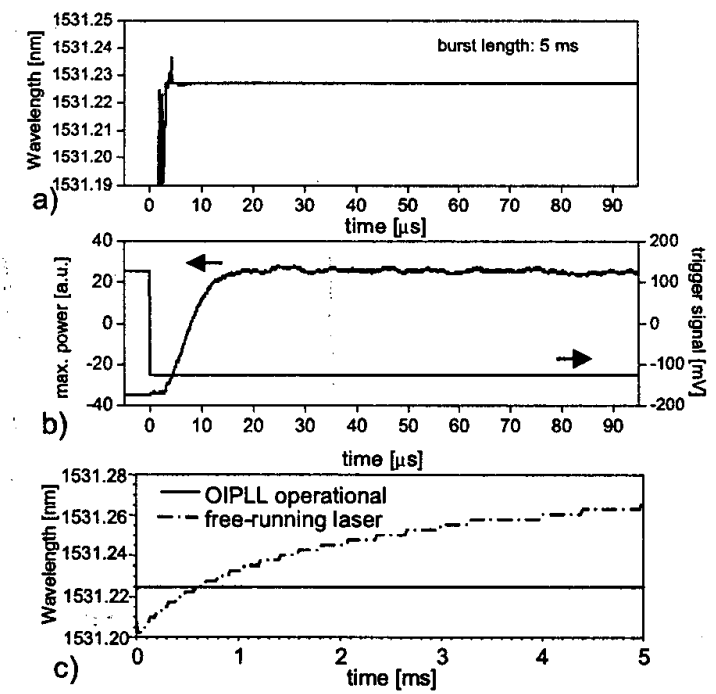

Fig. 3: Channel-hopping transient 1570-1532nm (resolution $1 \mathrm{pm}$ ): Wavelength stabilization is achieved within $5 \mu$ s of the onset of the electrical trigger signal (a), whilst the optical output power stabilizes within $15 \mu \mathrm{s}$ (b). The OIPLL removes any wavelength drift (solid line) as compared to the unlocked tuneable laser (dash-dot line) (c).

In summary, all fibre-based OIPLL circuits were used with widely tuneable lasers to create an optical frequency sythesiser offering zero-frequency-error relative to the reference signals supplied. Use of the OIPLL technique enables wide locking ranges $(>80 \mathrm{GHz})$ to be obtained with wide $(>10 \mathrm{MHz})$ linewidth slave lasers, without the need for short loop delay optics or electronics. Channel frequency errors remained below the measurement limit of 1 $\mathrm{kHz}$ while laser chip temperature was tuned over a $5 \mathrm{~K}$ range. Furthermore the side mode suppression ratio remained better than $35 \mathrm{~dB}$. Finally, wavelength hopping times of $<5 \mu$ s were obtained, without subsequent thermal equilibration drift. Such sources are likely to find application in metrology for existing DWDM networks and for future burst routed DWDM networks. Future work will concentrate on increasing the resolution and speed of the channel hopping test system to determine the ultimate hopping speed of the transmitter (expected to be in the ns region [2]).

[1] B. Cai, D. Wake, A. J. Seeds, "Microwave frequency synthesis using injection locked laser comb line selection," paper WD2 IEEE LEOS Summer topical meeting on RF opto-electronics, 1995.

[2] A. C. Bordonalli and A. J. Seeds, "High-performance phase locking of wide linewidth semiconductor lasers by combined use of optical injection locking and optical phase-lock loop," J. Lightwave Technol., 17, 328-342 (1999).

[3] C. F. C. Silva et al, "Terahertz span > 60-channel exact frequency dense WDM source using comb generation and SG-DBR injection-locked laser filtering," IEEE Photon. Technol. Lett., 13, 370-372 (2001). 